\title{
Hyperintense Vessels on FLAIR: Hemodynamic Correlates and Response to Thrombolysis
}

\author{
A. Kufner, I. Galinovic, V. Ambrosi, C.H. Nolte, M. Endres, J.B. Fiebach, and M. Ebinger
}

\begin{abstract}
BACKGROUND AND PURPOSE: Hyperintense vessels on baseline FLAIR MR imaging of patients with ischemic stroke have been linked to leptomeningeal collateralization, yet the ability of these to maintain viable ischemic tissue remains unclear. We investigated whether hyperintense vessels on FLAIR are associated with the severity of hypoperfusion and response to thrombolysis in patients treated with intravenous tissue-plasminogen activator.
\end{abstract}

MATERIALS AND METHODS: Consecutive patients with ischemic stroke with an MR imaging before and within 24 hours of treatment, with proved vessel occlusion and available time-to-maximum maps were included $(n=62)$. The severity of hypoperfusion was characterized on the basis of the hypoperfusion intensity ratio (volume with severe/mild hypoperfusion [time-to-maximum $\geq 8$ seconds / time-to-maximum $\geq 2$ seconds]). The hypoperfusion intensity ratio was dichotomized at the median to differentiate moderate (hypoperfusion intensity ratio $\leq 0.447$ ) and severe (hypoperfusion intensity ratio $>0.447$ ) hypoperfusion. Good outcome was defined as a modified Rankin Scale score of $\leq 2$.

RESULTS: Hyperintense vessels on FLAIR were identified in 54 patients (87\%). Patients with extensive hyperintense vessels on FLAIR ( $>4$ sections) had higher NIHSS scores, larger baseline lesion volumes, higher rates of perfusion-diffusion mismatch, and more severe hypoperfusion (hypoperfusion intensity ratio). In stepwise backward multivariate regression analysis for the dichotomized hypoperfusion intensity ratio (including stroke etiology, age, perfusion deficit, baseline lesion volume, smoking, and extent of hyperintense vessels on FLAIR), extensive hyperintense vessels on FLAIR were independently associated with severe hypoperfusion (OR, 6.8; 95\% Cl, 1.1-42.7; $P=.04$ ). The hypoperfusion intensity ratio was an independent predictor of a worse functional outcome at 3 months poststroke $(\mathrm{OR}, 0.2 ; 95 \% \mathrm{Cl}, 0.5-0.6 ; P<.01)$.

CONCLUSIONS: Hyperintense vessels on FLAIR are associated with larger perfusion deficits, larger infarct growth, and more severe hypoperfusion, suggesting that hyperintense vessels on FLAIR most likely indicate severe ischemia as a result of insufficient collateralization.

ABBREVIATIONS: $\mathrm{FHV}=$ hyperintense vessels on $\mathrm{FLAIR} ; \mathrm{HIR}=$ hypoperfusion intensity ratio; $\mathrm{IQR}=$ interquartile range; $\mathrm{Tmax}=$ time-to-maximum; $\mathrm{TOAST}=$ Trial of Org 10172 in Acute Stroke Treatment

W hile most studies agree that hyperintense vessels on FLAIR (FHV) are highly associated with large-vessel occlusion, results on the underlying pathophysiology are seemingly split. ${ }^{1}$ Several studies suggest FHV to be indicative of hemodynamic stress, inadequate collateralization, and poor functional recovery. ${ }^{2-5}$ Conversely, others attributed FHV to increased leptomen-

Received September 23, 2014; accepted after revision January 8, 2015.

From the Klinik und Hochschulambulanz für Neurologie (A.K., C.H.N., M. Endres, M. Ebinger), International Graduate Program Medical Neurosciences (A.K., V.A.), and Cluster of Excellence NeuroCure (M. Endres), Charité-Universitätsmedizin Berlin, Berlin, Germany; and Center for Stroke Research Berlin (I.G., C.H.N., M. Endres, J.B.F., M. Ebinger), Berlin, Germany.

This work was funded by the Federal Ministry of Education and Research via the grant Center for Stroke Research Berlin (01 EO 0801), the Volkswagen Foundation (Lichtenberg program to Matthias Endres), the EU (EUSTROKE, ARISE), and the German Research Foundation (NeuroCure, SFB-TR 43).

ingeal collateralization and found an association with smaller lesions, slower infarct progression, and better prognosis. ${ }^{6-10}$

The apparent contradiction of these studies may stem from the use of different methodologies and the diversity of populations studied, making the plethora of results challenging to analyze. Nevertheless, 2 critical questions remain due to lack of comprehensive imaging data and long-term clinical follow-up: Do FHV represent good collateralization or indicate the insufficiency of established collaterals to maintain ischemic tissue? Second, does FHV have clinical relevance in terms of functional recovery?

Please address correspondence to Anna Kufner, MSc, Klinik und Hochschulambulanz für Neurologie, Charité-Universitätsmedizin Berlin, Charitéplatz 1, Berlin-10117, Germany; e-mail: anna.kufner@charite.de

- Indicates open access to non-subscribers at www.ajnr.org

http://dx.doi.org/10.3174/ajnr.A4320 
The difficulty in determining collateral status poses a major challenge in answering these questions because digital subtraction angiography is not always readily available and associated risks may not be justified in most cases. Bang et $\mathrm{al}^{11}$ used a hypoperfusion intensity ratio (HIR: time-to-maximum [Tmax] $\geq 8$ seconds/Tmax $\geq 2$ seconds) on baseline MR perfusion imaging as a surrogate marker of collateral status and found that excellent and intermediate collateral grades were highly associated with lower HIRs.

In the PRE-FLAIR study, patients with FHV had larger initial lesion volumes and more severe clinical impairment ${ }^{12}$; therefore, we hypothesized that patients with FHV would have more severe hypoperfusion, suggesting the insufficiency of established collaterals to maintain ischemic tissue before reperfusion is achieved. In this study, we investigated whether the extent of FHV is correlated with the severity of hypoperfusion by using Tmax perfusion maps and whether this plays a role in response to thrombolysis in patients with arterial occlusion treated with intravenous recombinant tissue plasminogen activator.

\section{MATERIALS AND METHODS}

This was a retrospective study conducted at the Center for Stroke Research Berlin at Charité University Hospital. Consecutive patients with acute stroke recruited between March 2008 and December 2012 (1000Plus study registered with clinicaltrials.gov; NCT 00715533) were selected for analysis on the basis of the following criteria: received IV-tPA within 4.5 hours of symptom onset, had MR imaging before and within 24 hours after treatment, and had proved vessel occlusion and acute perfusion maps of sufficient quality to postprocess into Tmax maps. MR imaging (3T, Tim Trio; Siemens, Erlangen, Germany) protocol included $\mathrm{T}^{*}$, FLAIR $(\mathrm{TE}=100 \mathrm{~ms}, \mathrm{TR}=8000 \mathrm{~ms}$, TI $=2370.5 \mathrm{~ms}$, $\mathrm{FOV}=220 \mathrm{~mm}$, matrix $=256 \times 232,5-\mathrm{mm}$ section thickness with a $0.5-\mathrm{mm}$ intersection gap), diffusion-weighted imaging $(\mathrm{TE}=93.1 \mathrm{~ms}, \mathrm{TR}=7600 \mathrm{~ms}, \mathrm{FOV}=230 \mathrm{~mm}$, matrix $=192 \times$ 192, 2.5-mm section thickness with no intersection gap), MR angiography $(\mathrm{TE}=3.86 \mathrm{~ms}, \mathrm{TR}=22.0 \mathrm{~ms}, \mathrm{FOV}=200 \mathrm{~mm}$, matrix $=384 \times 268,0.65-\mathrm{mm}$ section thickness with a $7.2 \mathrm{~mm}$ intersection gap), and perfusion imaging ( $\mathrm{TE}=29 \mathrm{~ms}, \mathrm{TR}=1390$ $\mathrm{ms}, \mathrm{FOV}=230 \mathrm{~mm}$, matrix $=128 \times 128,5-\mathrm{mm}$ section thickness with a $0.5-\mathrm{mm}$ intersection gap).

The following cerebrovascular risk factors were evaluated on admission by using previously published definitions ${ }^{13}$ : smoking, arterial hypertension, diabetes mellitus, hypolipoproteinemia, and atrial fibrillation. Stroke severity was assessed by using the National Institutes of Health Stroke Scale. Stroke etiology was determined by using the Trial of Org 10172 in Acute Stroke Treatment (TOAST) criteria. ${ }^{14}$ Functional outcome was assessed 3 months poststroke by using the modified Rankin Scale score; favorable outcome was defined as mRS of $\leq 2$.

Blinded to acute perfusion imaging, 2 raters (A.K., I.G.) independently counted the number of FLAIR sections with FHV, defined as focal, linear, or serpentine hyperintensities distal to the main occluded artery. In case of disagreement, raters met for consensus. The number of sections with FHV was dichotomized at the median ( $\leq$ or $>4$ sections) to distinguish between less pronounced versus more extensive FHV.
Postprocessing of DWI was performed by using MRIcro (Advanced Brain Imaging; McCausland Center for Brain Imaging, Columbia, South Carolina) to assess lesion volumes on acute and second-scan images to determine infarct growth (DWI lesion volume after thrombolysis - DWI baseline lesion volume). Stroketool (Digital Image Solutions; Frechen, Germany) was used to assess acute perfusion deficit volume (MTT $>6$ seconds). Perfusion-diffusion mismatch was assessed as a binary variable defined as follows: (perfusion deficit - DWI lesion volume)/DWI lesion volume $\times 100>20 \%$. Tmax maps of acute perfusion imaging images were created in Stroketool. The severity of the hypoperfusion was characterized on the basis of HIR (Fig 1), defined as tissue volume with severe hypoperfusion (Tmax $\geq 8$ seconds) divided by volume with mild hypoperfusion (Tmax $\geq 2$ seconds). HIR was dichotomized at the median to differentiate moderate $($ HIR $\leq 0.447)$ and severe (HIR $>0.447)$ hypoperfusion.

Arterial occlusion was evaluated on time-of-flight MRA. Arterial occlusions were categorized on the basis of the size of the occluded vessel: large-vessel occlusions (internal carotid artery and carotid-T occlusions), medium-vessel occlusions (ie, M1, M2, P1, P2, and vertebral occlusions), and small-vessel occlusions (ie, occlusion in distal arterial branches). ${ }^{15}$ Recanalization was defined as an increase in at least 2 Thrombolysis in Myocardial Infarction points within 24 hours of treatment based on acute and follow-up MRA.

For all 2-group analyses, we used the Fisher exact test and Mann-Whitney $U$ test as appropriate. We chose a stepwise backward regression model with a binary dependent variable (dichotomized HIR and outcome) for multivariate regression analyses; all parameters that reached a significance of $<.1$ in univariate analysis based on the dependent variable were included for analysis.

Stepwise backward multivariate regression analysis was performed to determine independent predictors of the severity of hypoperfusion. We included the following parameters: TOAST criteria, the size of the vessel occlusion, smoking, baseline perfusion deficit, baseline lesion volume, and extent of FHV. Analogously, stepwise backward regression analysis was performed for favorable outcome, with the only change being that age was also dichotomized at older than 70 years to increase external comparability $^{13}$ (age [older than 70 years], smoking, atrial fibrillation, HIR [>0.447], NIHSS score on admission, baseline lesion volume, and extent of FHV [ $>4$ sections]). All statistical analyses were performed by using SPSS, Version 19 (IBM, Armonk, New York).

\section{RESULTS}

Sixty-two patients were included for final analysis (mean age, $71.4 \pm 13.9$ years; $48.4 \%$ female; median NIHSS score on admission, 11 ; interquartile range [IQR], $4.8-16$ ); $87 \%$ had visible FHV on acute FLAIR $(n=54)$. Interrater reliability for the presence of FHV was high $(\kappa=0.86 ; P<.01)$; in 9 cases, raters differed in $>2$ sections when counting the number of sections with FHV.

Patients with extensive FHV ( $>4$ sections) had higher NIHSS scores on admission, larger baseline lesion volumes, higher rates of perfusion-diffusion mismatch, and more severe hypoperfusion (ie, HIR) (Table 1). Patient groups also differed in terms of stroke 

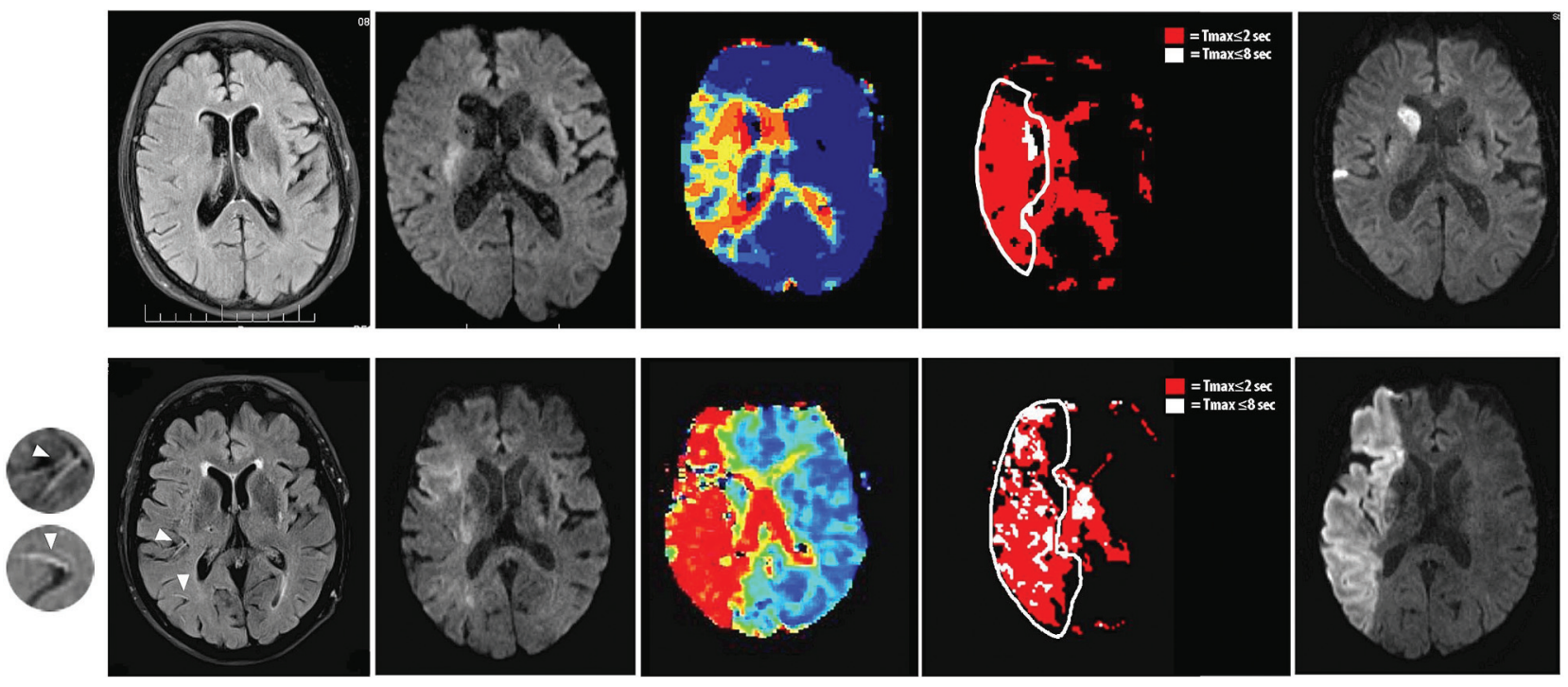

FIG 1. MR imaging (left-to-right: acute FLAIR, acute DWI, acute Tmax, dichotomized Tmax, follow-up DWI) of patients with middle cerebral artery occlusions (M1). Patient A (82 years of age; baseline NIHSS score, 13; baseline lesion volume, $1.4 \mathrm{~mL}$; HIR, 0.031; recanalization to Thrombolysis in Myocardial Infarction 2 following treatment; absolute infarct growth, $3.3 \mathrm{~mL}$ ) had FLAIR hyperintense vessels on 3 sections (FHV $\leq 4$; not visible on the depicted section) and patient B (76 years of age; baseline NIHSS score, 23; baseline lesion volume, $63.8 \mathrm{~mL}$; HIR, 0.53 ; no recanalization following treatment [Thrombolysis in Myocardial Infarction, 0]; absolute infarct growth, $80.2 \mathrm{~mL}$ ) had FHV on 8 sections (arrows; $\mathrm{FHV}>4)$.

Table 1: Comparison of baseline parameters and response to thrombolysis based on the number of sections with FLAIR hyperintense vessels ${ }^{a}$

\begin{tabular}{|c|c|c|c|}
\hline & $\begin{array}{c}\text { Visible FHV } \\
\text { on } \leq 4 \text { Sections }\end{array}$ & $\begin{array}{c}\text { Visible FHV } \\
\text { on }>4 \text { Sections }\end{array}$ & $P$ Value \\
\hline No. & 30 & 32 & \\
\hline Age (yr) (mean) (SD) & $71(10.8)$ & $71.9(16.5)$ & .42 \\
\hline Time to MRI in min (median) (IQR) & $88.5(72-103)$ & $93.5(61-121)$ & .98 \\
\hline NIHSS score on admission (median) (IQR) & $5(4-13)$ & $14.5(11-18)$ & $<.001$ \\
\hline NIHSS score on day 2 (median) (IQR) & $3(1-5)$ & $5(2-17)$ & .03 \\
\hline TOAST & & & .05 \\
\hline Cardioembolic (\%) (No.) & $40.7(11)$ & $36.7(11)$ & \\
\hline Macroangiopathic (\%) (No.) & $37(10)$ & $63.3(19)$ & \\
\hline Microangiopathic (\%) (No.) & $0(0)$ & $0(0)$ & \\
\hline Other (\%) (No.) & $7.4(2)$ & $0(0)$ & \\
\hline Competing causes (\%) (No.) & $14.8(4)$ & $0(0)$ & \\
\hline Baseline lesion volume (mL) (median) (IQR) & $1.6(.3-4.9)$ & $8.1(2.4-20.6)$ & $<.01$ \\
\hline Infarct growth (median) (IQR) & $1.2(0.2-12.7)$ & $12.8(2.5-43.9)$ & $<.01$ \\
\hline Perfusion-diffusion mismatch (\%) (No.) & $67.7(21)$ & $96.9(31)$ & $<.01$ \\
\hline Baseline perfusion deficit (mL) (median) (IQR) & $24.8(14.5-54.6)$ & $118(82-175)$ & $<.001$ \\
\hline $\operatorname{Tmax} \geq 2 \mathrm{sec}$ & $30(16.7-97.1)$ & $155(115.7-191.2)$ & $<.001$ \\
\hline $\operatorname{Tmax} \geq 8 \mathrm{sec}$ & $10.3(3.7-27.2)$ & $87.2(62.5-123.7)$ & $<.001$ \\
\hline HIR & $0.28(0.07-0.47)$ & $0.61(0.44-0.73)$ & $<.01$ \\
\hline Vessel occlusion size: & & & .29 \\
\hline Large (\%) (No.) & $20(6)$ & $9.4(3)$ & \\
\hline Medium (\%) (No.) & $80(24)$ & $90.6(29)$ & \\
\hline Small (\%) (No.) & $0(0)$ & $0(0)$ & \\
\hline Recanalization (\%) (No.) & $53.3(16)$ & $74.2(23)$ & .11 \\
\hline Favorable outcome at 3 months (\%) (No.) & $66.7(20)$ & $38.7(12)$ & .02 \\
\hline
\end{tabular}

${ }^{a}$ A Fisher exact test and Mann-Whitney $U$ test were used for categoric and continuous variables, respectively.

baseline lesion volumes, and lower rates of a favorable outcome (Table 2). Stroke etiology based on the TOAST criteria did not differ between groups.

In multivariate regression analysis for HIR, the extent of FHV was associated with severe hypoperfusion (OR, 6.8 ; 95\% CI, 1.1-42.7; $P=.04$; Table 3). In multivariate regression analysis for outcome, only baseline lesion volume (OR, 0.95; 95\% CI, 0.9-1.0; $P=.05$ ) and dichotomized HIR (OR, 0.17; 95\% CI, 0.05-0.57; $P<.01)$ remained in the model (Table 4). Last-step multivariate $r^{2}$ increased from 0.42 to 0.49 when age was included in the model as a continuous variable.

\section{DISCUSSION}

The extent of FHV is not only associated with larger perfusion deficits ${ }^{1,16}$ but is also an independent predictor of more severe hypoperfusion, resulting in larger infarct growth. Although we found no independent association between the extent of FHV and functional recovery,

etiology, in which patients with extensive FHV more often had macroangiopathic strokes. Despite the similarity of vessel-occlusion size and recanalization rates, patients with extensive FHV had larger infarct growth and a worse functional recovery 3 months poststroke.

Patients with severe hypoperfusion were significantly older and less often smokers and had higher NIHSS scores on admission, more sections with FHV, larger perfusion deficits, higher the severity of hypoperfusion was independently associated with a worse functional recovery 3 months poststroke (Table 2).

Similar to results from a multicenter observational study, ${ }^{12}$ approximately $87 \%$ of patients with proved vessel occlusion had at least 1 section with FHV on acute examination. Patients with extensive FHV (>4 sections) had comparatively higher NIHSS scores on admission, more severe hypoperfusion, larger infarct growth, and a worse functional outcome 3 months poststroke. While patient 
Table 2: Univariate analysis comparing patients with moderate hypoperfusion ( $\mathrm{HIR} \leq \mathbf{0 . 4 4 7}$ ) with patients with severe hypoperfusion (HIR $>0.447)^{\mathrm{a}}$

\begin{tabular}{|c|c|c|c|}
\hline & Low HIR $(\leq 0.447)$ & High HIR (>0.447) & $P$ Value \\
\hline No. & 31 & 31 & \\
\hline Age (yr) (mean) (SD) & $67.5(13.2)$ & 75.4 (13.7) & .02 \\
\hline Female (\%) (No.) & $51.6(16)$ & $45.2(14)$ & .8 \\
\hline Arterial hypertension (\%) (No.) & $80.6(25)$ & $87.1(27)$ & .73 \\
\hline Diabetes mellitus (\%) (No.) & $16.1(5)$ & $25.8(8)$ & .53 \\
\hline Smoking (\%) (No.) & $38.7(12)$ & $9.7(3)$ & .02 \\
\hline Hypolipoproteinemia (\%) (No.) & $54.8(17)$ & $46.4(15)$ & .9 \\
\hline Atrial fibrillation (\%) (No.) & $35.5(11)$ & $45.2(14)$ & .6 \\
\hline No. of sections with FHV (median) (IQR) & $3(1-7)$ & $7(4-10)$ & $<.01$ \\
\hline NIHSS score on admission (median) (IQR) & $5(4-13)$ & 15 (11-18) & $<.001$ \\
\hline Baseline lesion volume (mL) (median) (IQR) & $1.5(0.27-5.8)$ & $6.7(2.3-28.2)$ & $<.01$ \\
\hline Infarct growth (median) (IQR) & $1.6(0.2-10.6)$ & $6.2(1.4-39.0)$ & $<.01$ \\
\hline Baseline perfusion deficit (mL) (median) (IQR) & $41.2(15.8-101.7)$ & $117.1(56.0-171.6)$ & $<.01$ \\
\hline $\operatorname{Tmax} \geq 2 \mathrm{sec}$ & $75.6(24.7-179.4)$ & $143.1(70.5-170.2)$ & .08 \\
\hline $\operatorname{Tmax} \geq 8 \mathrm{sec}$ & $10.5(3.7-61.9)$ & $88.2(43.7-122.6)$ & $<.001$ \\
\hline Vessel-occlusion size: & & & .47 \\
\hline Large (\%) (No.) & $19.4(6)$ & $9.7(3)$ & \\
\hline Medium (\%) (No.) & $80.6(25)$ & $90.3(28)$ & \\
\hline Small (\%) (No.) & $0(0)$ & $0(0)$ & \\
\hline Recanalization (\%) (No.) & 70 (21) & $58.1(18)$ & .43 \\
\hline Favorable outcome at 3 months (\%) (No.) & $75.9(22)$ & $32.3(10)$ & $<.01$ \\
\hline
\end{tabular}

${ }^{a}$ A Fisher exact test and Mann-Whitney $U$ test were used for categoric and continuous variables, respectively.

Table 3: Stepwise multivariate regression analysis for severity of hypoperfusion (dichotomized at median)

\begin{tabular}{lcccc} 
& $\begin{array}{l}\text { Odds } \\
\text { Ratio }\end{array}$ & $\mathbf{9 5 \%} \mathbf{C l}$ & $\boldsymbol{P}$ Value & Univariate $\boldsymbol{r}^{2}$ \\
\hline First step (multivariate $\boldsymbol{r}^{2}=0.68$ ) & & & & \\
$\quad$ Age & 1.2 & $1.0-1.3$ & .01 & 0.12 \\
TOAST & 0.76 & $0.15-4.2$ & .77 & 0.004 \\
Baseline lesion volume & 1.1 & $1.0-1.2$ & .04 & 0.12 \\
Baseline perfusion deficit & 1.0 & $0.99-1.0$ & .06 & 0.12 \\
Sections with FHV (>4) & 6.7 & $1.0-41.9$ & .04 & 0.26 \\
Smoking & 0.04 & $0.001-0.99$ & .05 & 0.14 \\
Last step (multivariate $\left.r^{2}=0.68\right)$ & & & & \\
Age & 1.2 & $1.0-1.3$ & .01 & \\
Baseline lesion volume & 1.1 & $1.0-1.2$ & .05 & \\
Baseline perfusion deficit & 1.01 & $0.9-1.0$ & .06 & \\
Smoking & 0.04 & $0.001-0.96$ & .05 & \\
Sections with FHV $(>4)$ & 6.8 & $1.1-42.7$ & .04 & \\
\hline
\end{tabular}

Table 4: Stepwise multivariate regression analysis for favorable outcome (mRS $\leq 2$ )

\begin{tabular}{lcccc}
\hline & $\begin{array}{l}\text { Odds } \\
\text { Ratio }\end{array}$ & $\mathbf{9 5 \%} \mathbf{C l}$ & $\boldsymbol{P}$ Value & Univariate $\boldsymbol{r}^{\mathbf{2}}$ \\
\hline First step (multivariate $\left.\boldsymbol{r}^{2}=0.42\right)$ & & & & \\
$\quad$ Age (older than 70 years) & 1.0 & $0.23-5.8$ & .95 & 0.0 \\
Smoking & 0.84 & $0.20-4.5$ & .83 & 0.02 \\
Atrial fibrillation & 0.41 & $0.10-1.6$ & .21 & 0.14 \\
Sections with FHV $(>4)$ & 0.89 & $0.2-3.9$ & .88 & 0.12 \\
HIR (>0.447) & 0.24 & $0.05-1.1$ & .07 & 0.24 \\
NIHSS score on admission & 0.93 & $0.83-1.0$ & .12 & 0.25 \\
Baseline lesion volume & 0.96 & $0.90-1.0$ & .12 & 0.21 \\
Last step (multivariate $\left.r^{2}=0.36\right)$ & & & & \\
$\quad$ HIR (>0.447) & 0.17 & $0.05-0.57$ & $<.01$ & \\
Baseline lesion volume & 0.95 & $0.90-1.0$ & .05 & \\
\hline
\end{tabular}

ing, this study found that low HIR (moderate hypoperfusion) was highly associated with good collateral grades. This finding is in line with our hypothesis that FHV are indicative of severe hypoperfusion and insufficient collateralization. These results stand in contrast to previous studies suggesting that FHV represent increased leptomeningeal collateralization $^{6,7,10,17}$; these studies, however, did not report perfusion status or clinical follow-up.

Peripheral collateralization is an inherent defense mechanism following proximal vessel occlusion; collaterals can prolong tissue viability if blood flow is sufficient to keep hypoperfusion moderate until reperfusion is achieved. Vessels become hyperintense on FLAIR when blood flow is so slow that there is a loss of the flow-void phenomenon. Here, we observe an independent association between the extent of FHV and severe hypoperfusion (Table 3); however, the link to collateral flow is not yet clear. On the one hand, one might imagine that slow flow in collaterals directly causes arterial hyperintensities on FLAIR, suggesting that FHV directly depict insufficient or sluggish flow in collateral pathways. ${ }^{7,8}$ On the other hand, vessel occlusion and subsequent ischemia cause cerebral swelling, which likely compresses distal collateral vessels, slowing blood flow. Regardless, FHV most likely reflect slow flow in distal collateral pathways distributed over a large ischemic area, resulting in larger ischemic lesions. Hence, this frequently observed MR imaging feature may be useful as a tool to assess the severity of hypoperfusion over an ischemic area as a result of poor collateralization.

Of note, patients with large-vessel occlusion had less pronounced FHV $(<4)$ and more moderate hypoperfusion (low HIR), though these did not reach the level of significance (Tables 1 and 2). Carotid occlusions are frequently the end result of chronic occlusive disease, and the recruitment of col-

groups based on FHV differed in terms of stroke etiology, TOAST criteria showed neither association with the severity of hypoperfusion nor functional recovery in univariate analysis.

Bang et $\mathrm{al}^{11}$ used the same Tmax HIR ratio to determine the severity of the perfusion deficit and found similar results in terms of NIHSS scores on admission and infarct volume. Most interest- laterals often happens before acute stroke takes place. Thus, one might imagine that the longer large-vessel disease has been in existence before the index event, the greater the likelihood of excellent flow in collaterals. These collaterals might maintain good flow without visible FHV; however, this is mere speculation. 
Although HIR was an independent predictor of functional recovery, we observed no independent associations of the extent of FHV and outcome (Table 4). A possible explanation may lie in the heterogeneity of vessel occlusion size observed in this cohort; an independent analysis including only patients with middle cerebral artery occlusions is suggested.

In multivariate analysis for outcome, baseline lesion volume remained in the model, accounting for $21 \%$ of the variance; this is to be expected because initial infarct volume may reflect collateral status. While FHV may be indicative of insufficient collateral flow, the clinical relevance of this MR imaging sign in terms of predicting response to treatment remains unclear. A study by Olindo et $\mathrm{al}^{9}$ found extensive FHV associated with smaller infarcts pre- and posttreatment, as well as a better prognosis in patients with MCA occlusion. The discordance of these results may lie in the use of different MR imaging units (1T versus 3T), the characteristics of populations studied, and the question at hand. In this study, we focused primarily on the acute hemodynamic correlates of FHV, which show a clear independent association with more severe hypoperfusion; the prognostic value of FHV remains unresolved.

Most interesting, smoking was independently associated with moderate hypoperfusion, suggesting better collateralization in patients with this risk factor. Bang et $\mathrm{al}^{11}$ also found an association between smoking status and low HIR. Studies have suggested that long-term smoking-induced atherosclerosis leads to increased collateralization, ${ }^{18}$ which may explain this observed phenomenon.

This study has limitations. Most important, it is a retrospective observational study. Furthermore, lack of DSA did not allow direct assessment of collateral grade; the use of HIR only partially compensated for this limitation. Finally, due to small numbers, this study runs the risk of type 2 errors, and multivariable regression analyses may lead to overfitting.

\section{CONCLUSIONS}

The extent of FHV on baseline MR imaging is an independent predictor of severe hypoperfusion in patients with acute ischemic stroke. FHV were highly associated with more severe strokes and larger infarct growth, suggesting that this frequently observed MR imaging feature most likely indicates severe ischemia due to the insufficiency of established collaterals to maintain ischemic tissue before recanalization is achieved.

Disclosures: Christian H. Nolte-UNRELATED: Consultancy: Bristol-Myers Squibb, Pfizer, Bayer Pharma; Payment for Lectures (including service on Speakers Bureaus): Pfizer, Boehringer Ingelheim; OTHER: payment for lectures and travel grants from Boeheringer-Ingelheim and Takeda-Pharma. Matthia Endres—RELATED: Grant: DFG: Excellence Cluster NeuroCure, ${ }^{\star}$ Federal Ministry of Education and Research: Center for Stroke Research, ${ }^{\star}$ EU: WakeUp, ${ }^{*}$ Volkswagen Foundation: Lichtenberg-Program*; UNRELATED: Board Membership: Bayer, Boehringer Ingelheim, Pfizer, BMS, MSD, Sanofi, Comments: Advisory Board; Grants/Grants Pending: DFG,* EU,* AstraZeneca, ${ }^{\star}$ Roche, ${ }^{\star}$ Sanofi, ${ }^{\star}$ Comments: DFG: SFB TR43, KFO 247, KFO 213, EU: ESN, Counterstroke (an EU-funded research project in which specialists collaborate to develop novel therapies to treat stroke); Payment for Lectures (including service on Speakers Bureaus): Astra Zeneca, Bayer, Boston Scientific, Bristol-Myers Squibb, Boehringer-Ingelheim, Ever, Glaxo Smith Kline, MSD, Novartis, Pfizer, Sanofi; OTHER: He reports the following consultancies, grants and/or payments for lectures including service on speakers bureaus: Astra Zeneca, Bayer, Berlin Chemie, BMS, Boehringer-Ingelheim, Glaxo Smith Kline, Novartis, Pfizer, Sanofi-Aventis, Trommsdorff; he holds a patent for the upregulation of type III endothelial cell nitric oxide synthase by HMG-CoA reductase inhibitors (US patent number 6,147,109). Jochen B. FiebachRELATED: Consulting Fee or Honorarium: Boehringer; UNRELATED: Board Membership: Lundbeck, Sygnis, Synarc, Comments: Head of Imaging Board of the Desmotoplase in Acute Ischemic Stroke Trial (DIAS) $3 \& 4$ and Imaging Board DIAS J; central reader AXIS 2 trial; central reader i.a. Thrombolysis trial; Consultancy: Lundbeck, Comments: DIAS 3\&4; Payment for Lectures (including service on Speakers Bureaus): Boehringer Ingelheim, Siemens, Comments: stroke imaging; OTHER: He reports the following board memberships, consultancies and/or payments for lectures including service on speakers bureaus: Boehringer-Ingelheim, Lundbeck, Siemens, Sygnis, Synarc. *Money paid to the institution.

\section{REFERENCES}

1. Azizyan A, Sanossian N, Mogensen MA, et al. Fluid-attenuated inversion recovery vascular hyperintensities: an important imaging marker for cerebrovascular disease. AJNR Am J Neuroradiol 2011;32:1771-75

2. Ebinger M, Kufner A, Galinovic I, et al. Fluid-attenuated inversion recovery images and stroke outcome after thrombolysis. Stroke 2012;43:539-42

3. Girot M, Gauvrit JY, Cordonnier C, et al. Prognostic value of hyperintense vessel signals on fluid-attenuated inversion recovery sequences in acute cerebral ischemia. Eur Neurol 2007;57:75-79

4. Hohenhaus M, Schmidt WU, Brunecker P, et al. FLAIR vascular hyperintensities in acute ICA and MCA infarction: a marker for mismatch and stroke severity? Cerebrovasc Dis 2012;34:63-69

5. Kamran S, Bates V, Bakshi R, et al. Significance of hyperintense vessels on FLAIR MRI in acute stroke. Neurology 2000;55:265-69

6. Huang X, Liu W, Zhu W, et al. Distal hyperintense vessels on FLAIR: a prognostic indicator of acute ischemic stroke. Eur Neurol 2012;68:214-20

7. Lee KY, Latour LL, Luby M, et al. Distal hyperintense vessels on FLAIR: an MRI marker for collateral circulation in acute stroke? Neurology 2009;72:1134-39

8. Liu W, Xu G, Yue X, et al. Hyperintense vessels on FLAIR: a useful non-invasive method for assessing intracerebral collaterals. Eur J Radiol 2011;80:786-91

9. Olindo S, Chausson N, Joux J, et al. Fluid-attenuated inversion recovery vascular hyperintensity: an early predictor of clinical outcome in proximal middle cerebral artery occlusion. Arch Neurol 2012;69:1462-68

10. Pérez de la Ossa N, Hernandez-Perez M, Domenech S, et al. Hyperintensity of distal vessels on FLAIR is associated with slow progression of the infarction in acute ischemic stroke. Cerebrovasc Dis 2012;34:376-84

11. Bang OY, Saver JL, Alger JR, et al. Determinants of the distribution and severity of hypoperfusion in patients with ischemic stroke. Neurology 2008;71:1804-11

12. Cheng B, Ebinger M, Kufner A, et al. Hyperintense vessels on acute stroke fluid-attenuated inversion recovery imaging: associations with clinical and other MRI findings. Stroke 2012;43:2957-61

13. Kufner A, Nolte CH, Galinovic I, et al. Smoking-thrombolysis paradox: recanalization and reperfusion rates after intravenous tissue plasminogen activator in smokers with ischemic stroke. Stroke 2013;44:407-13

14. Adams HP Jr, Bendixen BH, Kappelle LJ, et al; TOAST Investigators. Classification of subtype of acute ischemic stroke: definitions for use in a multicenter clinical trial. Stroke 1993;24:35-41

15. TIMI Study Group. The Thrombolysis in Myocardial Infarction (TIMI) trial-phase I findings. N Engl J Med 1985;312:932-36

16. Schellinger PD. Diagnostic accuracy and relevance of early MRI vessel signs are highly overrated. Cerebrovasc Dis 2006;21:215; author reply $215-16$

17. Liu W, Xu G, Yue X, et al. Hyperintense vessels on FLAIR: a useful non-invasive method for assessing intracerebral collaterals. Eur J Radiol 2011;80:786-91

18. Koerselman J, de Jaegere PP, Verhaar MC, et al. Coronary collateral circulation: the effects of smoking and alcohol. Atherosclerosis 2007; 191:191-98 